\title{
Synucleins Are Developmentally Expressed, and $\alpha$-Synuclein Regulates the Size of the Presynaptic Vesicular Pool in Primary Hippocampal Neurons
}

\author{
Diane D. Murphy, Susan M. Rueter, John Q. Trojanowski, and Virginia M.-Y. Lee \\ Center for Neurodegenerative Disease Research, Department of Pathology and Laboratory Medicine, University of \\ Pennsylvania Medical School, Philade/phia, Pennsylvania 19104
}

$\alpha-, \beta-$, and $\gamma$-Synuclein, a novel family of neuronal proteins, has become the focus of research interest because $\alpha$-synuclein has been increasingly implicated in the pathogenesis of Parkinson's and Alzheimer's disease. However, the normal functions of the synucleins are still unknown. For this reason, we characterized $\alpha-, \beta-$, and $\gamma$-synuclein expression in primary hippocampal neuronal cultures and showed that the onset of $\alpha$ - and $\beta$-synuclein expression was delayed after synaptic development, suggesting that these synucleins may not be essential for synapse formation. In mature cultured primary neurons, $\alpha$ - and $\beta$-synuclein colocalized almost exclusively with synaptophysin in the presynaptic terminal, whereas little $\gamma$-synuclein was expressed at all. To assess the function of $\alpha$-synuclein, we suppressed expression of this protein with antisense oligonucleotide technology. Morphometric ultrastructural analysis of the $\alpha$-synuclein antisense oligonucleotide-treated cultures revealed a significant reduction in the distal pool of synaptic vesicles. These data suggest that one function of $\alpha$-synuclein may be to regulate the size of distinct pools of synaptic vesicles in mature neurons.

Key words: Lewy bodies; Parkinson's disease; primary neurons; synapse; synuclein; vesicle
$\alpha$-Synuclein was first isolated in 1988 from synaptic vesicles of Torpedo californica and rat brain (Maroteaux et al., 1988). Subsequently, other members of this family of neuronal proteins have been identified: $\beta$-synuclein, also found mainly in the CNS, $\gamma$-synuclein, more abundant in the PNS than in the CNS, and synoretin, present primarily in the retina (Nakajo et al., 1990; Jakes et al., 1994; Ji et al., 1997; Buchman et al., 1998; Surguchov et al., 1999). $\alpha$-Synuclein has been shown to be a primary component of Lewy bodies, the neuropathological hallmarks of sporadic Parkinson's disease (PD) (Spillantini et al., 1997; Baba et al., 1998), and pathogenic $\alpha$-synuclein gene mutations have been identified in familial forms of PD (Polymeropoulos et al., 1997; Kruger et al., 1998). Moreover, $\alpha$-synuclein has been detected in Lewy bodies that are diagnostic of the Lewy body variant of Alzheimer's Disease (LBVAD) as well as dementia with Lewy bodies (DLB) (Spillantini et al., 1997; Baba et al., 1998; Trojanowski et al., 1998). Thus, insight into mechanisms of synuclein pathology may be critical for understanding brain degeneration in PD, LBVAD, and DLB (Trojanowski et al., 1998).

The implication of $\alpha$-synuclein in neurodegenerative disease has stimulated efforts to elucidate the normal distribution and functions of $\alpha-, \beta$-, and $\gamma$-synuclein. For example, because $\alpha$-synuclein is localized to presynaptic terminals throughout the adult mammalian brain and may appear earlier than synaptophy-

\footnotetext{
Received Nov. 11, 1999; revised Feb. 14, 2000; accepted Feb. 24, 2000.

This work was supported by grants from the National Institute on Aging. We acknowledge Drs. N. B. Cole, R.W. Doms, and V. Zhukareva for scientific contributions, Dr. R. Balice-Gordon for use of the confocal microscope, and N. Shah and J. Sanzo of the electron microscopy facility for EM preparations and use of the electron microscope.

D.M. and S.R. contributed equally to this work.

Correspondence should be addressed to Dr. Virginia M.-Y. Lee, Department of Pathology and Laboratory Medicine, University of Pennsylvania School of Medicine, Maloney 3, HUP, 3600 Spruce Street, Philadelphia, PA 19104-4283. E-mail: vmylee@mail.med.upenn.edu.

Copyright (C) 2000 Society for Neuroscience $\quad 0270-6474 / 00 / 203214-07 \$ 15.00 / 0$
}

sin during CNS development, it has been suggested to play a role in synaptogenesis (Hsu et al., 1998). However, in vitro studies show that the vesicle-associated protein synapsin I appears as early as $24 \mathrm{hr}$, whereas $\alpha$-synuclein is not detected until day 5 (Withers et al., 1997). Thus, it is unclear whether $\alpha$-synuclein is needed for the development of synapses or for the maturation and modulation of previously existing ones. There is also evidence that $\alpha$-synuclein may associate with synaptic vesicles because $\alpha$-synuclein has been detected in vesicular fractions of human brain (Irizarry et al., 1996) and binds to both synthetic membranes (Davidson et al., 1998) and vesicles isolated from rat brain (Jensen et al., 1998).

The present study assessed $\alpha$-, $\beta$-, and $\gamma$-synuclein expression in primary hippocampal neurons grown at a substantially increased density. These high-density cultures exhibit a robust, rapid development of synaptic contacts and spines (Papa et al., 1995) that corresponds to those seen during postnatal hippocampal development. Moreover, the synaptic ultrastructure of high-density cultures resembles that found in situ (Bartlett and Banker, 1984). We used light microscopic immunocytochemistry, electron microscopy, and protein biochemistry to characterize the expression of the synuclein family of proteins in hippocampal neurons up to 3 weeks in culture. We also examined changes in synaptic structure after treatment of the neurons with antisense (AS) oligonucleotides to reduce $\alpha$-synuclein expression. Here, we report that $\alpha$ - and $\beta$-synuclein were expressed after synaptophysin and localized almost exclusively to presynaptic terminals of mature neurons. In addition, downregulation of $\alpha$-synuclein by AS oligonucleotides caused a selective reduction in the size of the presynaptic vesicular pool. Our data suggest that $\alpha$-synuclein may interact with and regulate specific pools of synaptic vesicles, thereby modulating synaptic functions in the normal brain. These findings have important implications for the role $\alpha$-synuclein may play in neurodegenerative disease. 

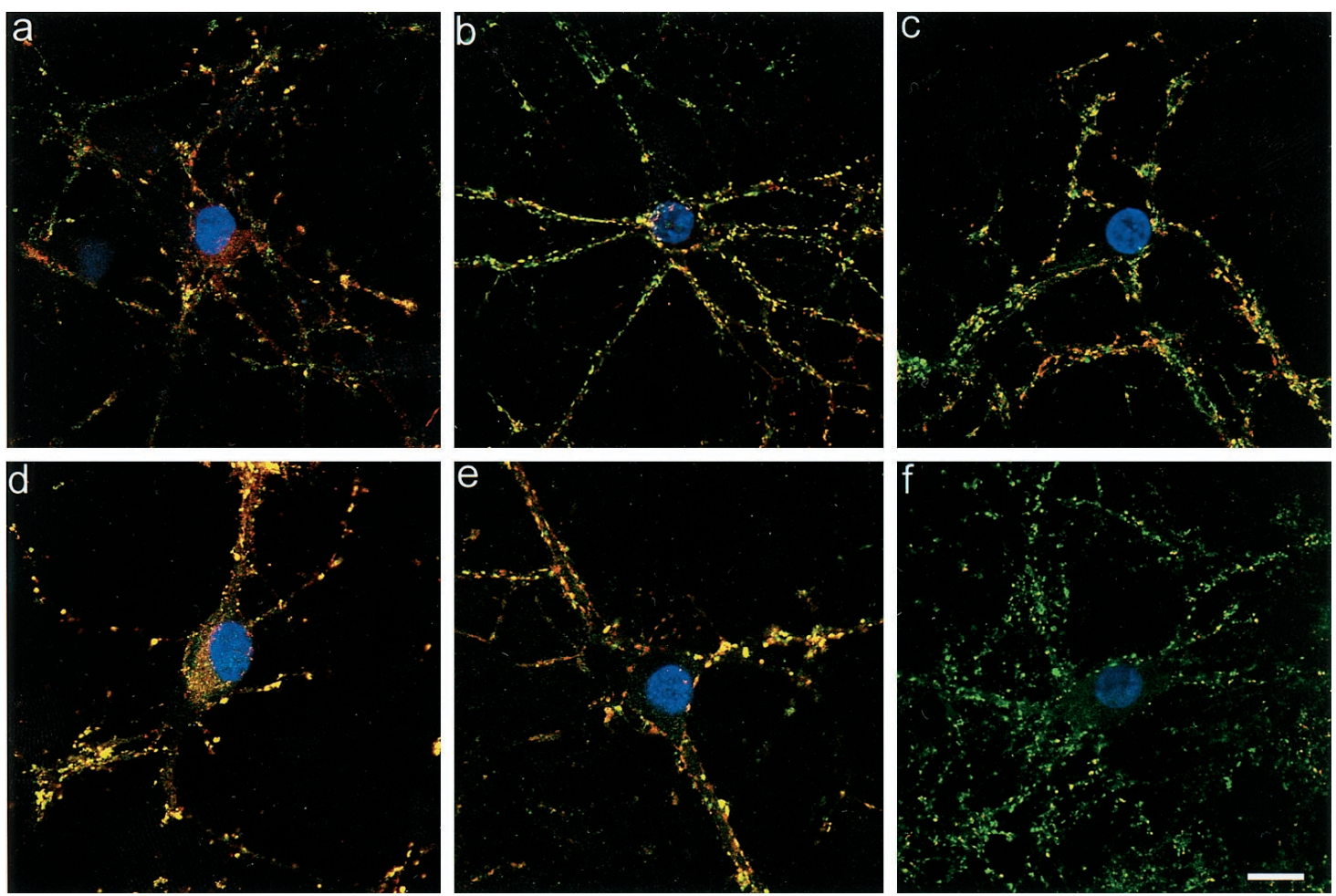

Figure 1. Synuclein characterization by immunofluorescence. $1(a)-, 2(b)$-, and 3 (c)-week-old cultures stained for $\alpha$-synuclein (red) and synaptophysin (green). Cytosolic $\alpha$-synuclein staining is evident in $a . d$, Double labeling of $\alpha$-synuclein ( green) and GAD (red) show colocalization in an inhibitory neuron and in GABAergic presynaptic terminals (yellow). $e, \alpha$ - and $\beta$-synuclein are colocalized (yellow) to the presynaptic terminal. $f$, $\gamma$-synuclein (red) is poorly expressed in mature hippocampal neurons as compared to synaptophysin (green). The nuclei in $a-f$ are labeled by Hoechst (blue) dye. Scale bar, $10 \mu \mathrm{m}$.

\section{MATERIALS AND METHODS}

Hippocampal cultures. High-density hippocampal neuronal cultures were prepared as previously described (Papa et al., 1995). Briefly, 20-d-old embryos were taken from anesthetized Sprague Dawley rats. The brains were removed and placed in ice-cold $\left(4^{\circ} \mathrm{C}\right) \mathrm{L}-15$ medium supplemented with $0.6 \%$ glucose and $15 \mu \mathrm{g} / \mathrm{ml}$ gentamicin. The hippocampus was dissected and mechanically disaggregated by gentle trituration using a Pasteur pipette. Dissociated cells (500,000 cells/well) were plated onto sterile $12 \mathrm{~mm}$ glass or Thermanox coverslips that were coated with collagen $(50 \mu \mathrm{g} / \mathrm{ml})$ and poly-L-lysine $(15 \mu \mathrm{g} / \mathrm{ml})$. Cells were also prepared on six-well tissue culture dishes for Western blot analysis. Thermanox coverslips were used for electron microscopy (see below). The plating medium was Eagle's MEM containing 5\% heat-inactivated horse serum, $5 \%$ fetal calf serum, $2 \mathrm{~mm}$ glutamine, $0.6 \%$ glucose, and $15 \mu \mathrm{g} / \mathrm{ml}$ gentamicin. Cells were incubated at $37^{\circ} \mathrm{C}$ with $5 \% \mathrm{CO}_{2}$. The first change of medium, $\sim 4-6 \mathrm{~d}$ after plating, included $50 \mu \mathrm{g} / \mathrm{ml}$ uridine and 20 $\mu \mathrm{g} / \mathrm{ml}$ deoxyuridine to prevent glial cell overgrowth. The cultures were fed thereafter 1-2 times a week, with Eagle's MEM as above.

Immunocytochemistry. Cells were fixed in $4 \%$ paraformaldehyde in PBS. After blocking and permeabilization in 5\% goat serum with $0.1 \%$ saponin, cells were incubated with primary antibodies to the following: Syn102 (recognizes $\alpha$ and $\beta$ synuclein), SNL-1 ( $\alpha$-synuclein-specific), syn207 ( $\beta$-synuclein-specific) (Tu et al., 1998), and a rabbit polyclonal antiserum specific for $\gamma$-synuclein, VAMP (Chemicon, Temecula, CA), synaptophysin (Boehringer Mannheim, Mannheim, Germany), syntaxin (Upstate Biotechnology, Lake Placid, NY), synapsin I (Molecular Probes, Eugene, OR), and MAP2 (Upstate Biotechnology). The antisynuclein antibodies used here were previously characterized, and the specificity of each antibody for $\alpha-, \beta$-, and $\gamma$-synuclein was determined (Giasson et al., 2000). Cells were washed and incubated with FITC- or rhodamine-conjugated secondary antibodies. Cells were then imaged on a Leica (Nussloch, Germany) confocal laser-scanning microscope, using a 1.4 NA, $100 \times$ oil immersion objective lens.

Western blot analysis. Total protein extracts were prepared from 6-well dishes of primary hippocampal neurons in detergent-containing cell extraction buffer (50 mM Tris $\mathrm{HCl}$, pH 7.5, 1 mm EDTA, $150 \mathrm{~mm} \mathrm{NaCl}$,
$0.1 \%$ Triton $\mathrm{X}-100,0.5 \mathrm{~mm}$ PMSF, and $1 \mathrm{~mm}$ DTT, protease inhibitor cocktail). Harvested cells were spun at $100,000 \times g$ in a TL100 ultracentrif uge (Beckman). Protein concentration in the extracts was determined by the Bradford assay. Approximately $30 \mu \mathrm{g}$ of the supernatant was fractionated by SDS-PAGE and transferred onto nitrocellulose. The blots were incubated in blocking solution containing 5\% nonfat dry milk in $1 \times$ Tris-buffered saline (TBS) and then incubated overnight at $4^{\circ} \mathrm{C}$ with the various primary antibodies described above diluted in 5\% milk/TBS. As a control, blots were also stained for neuron-specific enolase (NSE; Polysciences, Warrington, PA) to normalize the amount of neuronal protein. The blots were washed in TBS-T (TBS $+0.1 \%$ Tween 20) and incubated for $2 \mathrm{hr}$ at room temperature with secondary antibodies $\left[{ }^{125} \mathrm{I}\right]$ conjugated to either anti-mouse IgG or Protein A at a concentration of $1 \mu \mathrm{Ci} / \mathrm{ml}$ of PTX buffer $(10 \mathrm{~mm}$ sodium phosphate, $\mathrm{pH}$ 7.3, 1 mm EDTA, $150 \mathrm{~mm} \mathrm{NaCl}, 0.2 \%$ Triton $\mathrm{X}-100$, and $4 \%$ BSA). The blots were washed again with TBS-T and exposed in a PhosphorImager cassette (Molecular Dynamics, Sunnyvale, CA) for 24-48 hr. The protein levels were quantified using ImageQuant software (Molecular Dynamics), each lane normalized to NSE levels.

Antisense treatments. Oligonucleotides were prepared from the $5^{\prime}$ end of the coding region for rat $\alpha$-synuclein. The antisense (AS) oligonucleotide sequence was $5^{\prime}$-CCTTTCATGAACAC ATCCATGGC-3'. The reverse sense $(\mathrm{S})$ oligonucleotide sequence (5'-GCCATGGA TGTGTTCATGAAAGG-3') and a scrambled oligonucleotide sequence $\left(5^{\prime}\right.$ TAGCTCGCTACGTAATCACCACT-3') served as controls. All oligonucleotides contained a phosphorothioate group at every residue. Cells were placed in N3 serum-free media (Banker and Goslin, 1991) on the day before and during AS oligonucleotide treatments to provide better delivery of oligonucleotides to the cells. Cells [6 $\mathrm{d}$ in vitro (div)] received $5 \mu \mathrm{M}$ AS or S oligonucleotide every other day for an additional $6 \mathrm{~d}$. To assay $\alpha$-synuclein involvement in synaptic development, an additional set of cultures were dosed at $3 \mathrm{div}$ and continuously for 2 weeks thereafter, and subsequently stained for synaptophysin.

Electron microscopy. Control, AS and S oligonucleotide-treated cells (four experiments in four separate sets of cultures) grown on Thermanox coverslips were fixed in modified Karnoversusky's fixative (Electron 
a

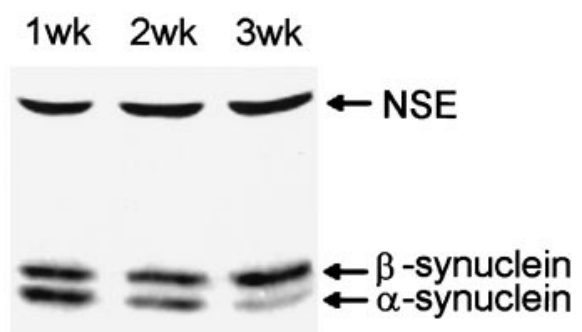

b

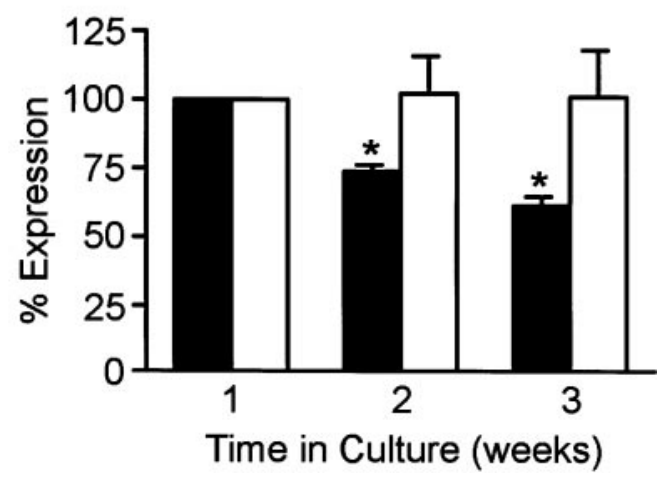

Figure 2. Synuclein protein expression in cultured hippocampal neurons. $\alpha$ - and $\beta$-synuclein expression in neurons at $1,2,3$ weeks in culture was determined by quantitative Western blot analysis from Triton X-100 soluble cell extracts $(a)$. The blots were probed with antibody syn102 (specific for both $\alpha$ - and $\beta$-synucleins) and an antibody to NSE. $b$, Quantitation of $\alpha$ - and $\beta$-synuclein expression at 1,2 , and 3 weeks in culture. Closed bars represent $\alpha$-synuclein expression, and open bars represent $\beta$-synuclein expression. All synuclein signals were normalized to the signal for NSE to account for the amount of neuronal protein loaded per lane. Results are presented as the average percentage of expression \pm SEM. The expression at 1 week was arbitrarily set at $100 \%$. $n=3 ;{ }^{*} p<0.05$.

Microscopy Sciences) consisting of 3\% paraformaldehyde and 2.5\% glutaraldehyde in $0.1 \mathrm{M}$ phosphate buffer. Cells were then dehydrated in graded series of ethanol and embedded in araldite. Thin sections were cut on an UltracutE, stained, and observed in a JEOL 100 microscope at $33,000 \times$. Synapses were selected using a modified protocol as previously described (Reist et al., 1998). Briefly, only those synapses with a welldefined postsynaptic density were included in the counting assay, and grids were scanned so as not to photograph the same synapse twice. Negatives were scanned and calibrated using NIH Image software. Cross-sectional area of each synapse was measured, and the number of vesicles counted as previously described (Pozzo-Miller et al., 1999).
Vesicles touching the synaptic membrane, or those within less than a vesicle diameter to the membrane were counted as "docked." The others were counted as the "distal" vesicular pool. Counts were divided by the cross-sectional area of the synapse, and means and SEs were calculated. Seventy-five, 97, and 56 synapses were counted for the control, antisense, and sense treatment groups, respectively.

\section{RESULTS}

The expression of $\alpha-, \beta$-, and $\gamma$-synuclein was monitored by indirect immunofluorescence from 2-21 div. While synaptophysin was observed within the Golgi complex and early synapses as soon as 3 div, $\alpha$ - and $\beta$-synuclein were not expressed until 6 div. At this time, $\alpha$-synuclein (Fig. $1 a$ ) and $\beta$-synuclein (data not shown) were detected primarily in the cytosol throughout the cell body and primary processes, but also in a few synapses. By $14 \operatorname{div}, \alpha$ - and $\beta$-synuclein were predominantly localized to the presynaptic terminal, as evidenced by colocalization with synaptophysin (a well characterized presynaptic protein), and at 3 weeks they appeared almost exclusively at the presynapse (Fig. 1b,c). At 3 weeks, these neurons are considered to be "mature" because they demonstrate a stable number of excitatory connections, dendritic outgrowth, and dendritic spine density that increase no further during the culture life span (Papa et al., 1995). The expression of $\alpha$ - and $\beta$-synuclein was not confined to a particular type of synapse, excitatory or inhibitory, because they colocalized with glutamic acid decarboxylase (GAD) which specifically labels inhibitory GABAergic synapses (Fig. $1 d$ ). Both $\alpha$ - and $\beta$-synuclein showed the same pattern of development and colocalized with one another (Fig. 1e). Because parallel studies revealed very few $\gamma$-synuclein-positive boutons at any time point (Fig. 1f), $\gamma$-synuclein was not included in further experiments.

Total $\alpha$ - and $\beta$-synuclein expression levels were also monitored by quantitative Western analysis at 1,2 , and 3 weeks in culture. Western analysis indicated that $\alpha$-synuclein expression peaked at 1 week and decreased by $\sim 25 \%$ at 2 weeks and $\sim 40 \%$ at 3 weeks. $\beta$-Synuclein expression levels did not change significantly over the same time period in culture (Fig. 2).

To probe the functional role of $\alpha$-synuclein in the presynaptic terminal, antisense (AS) oligonucleotides were engineered and delivered to the cells to prevent $\alpha$-synuclein expression. A sense (S) oligonucleotide was used as a control as were cells that received no oligonucleotide treatment and were grown in serumfree medium. Cells treated for up to 6 div with AS oligonucleotide displayed $\alpha$-synuclein immunofluorescence that was substantially reduced compared to control or $\mathrm{S}$ oligonucleotide-treated cultures (Fig. 3), however, MAP2 staining was unchanged, indicating that the AS oligonucleotide did not disrupt normal dendritic development (data not shown). Quantitative Western blots showed that $\alpha$-synuclein expression was decreased to $53 \pm 3.1 \%$ of controls by AS oligonucleotide treatment, whereas the levels of
Figure 3. A specific AS oligonucleotide decreases $\alpha$-synuclein expression. Cultured hippocampal neurons were either not treated $(a)$, or treated with AS oligonucleotide $(b)$ or with $\mathrm{S}$ oligonucleotide (c) for $6 \mathrm{~d}$ and immunostained with antibody SNL-1 (specific for $\alpha$-synuclein). Scale bar, $10 \mu \mathrm{m}$.
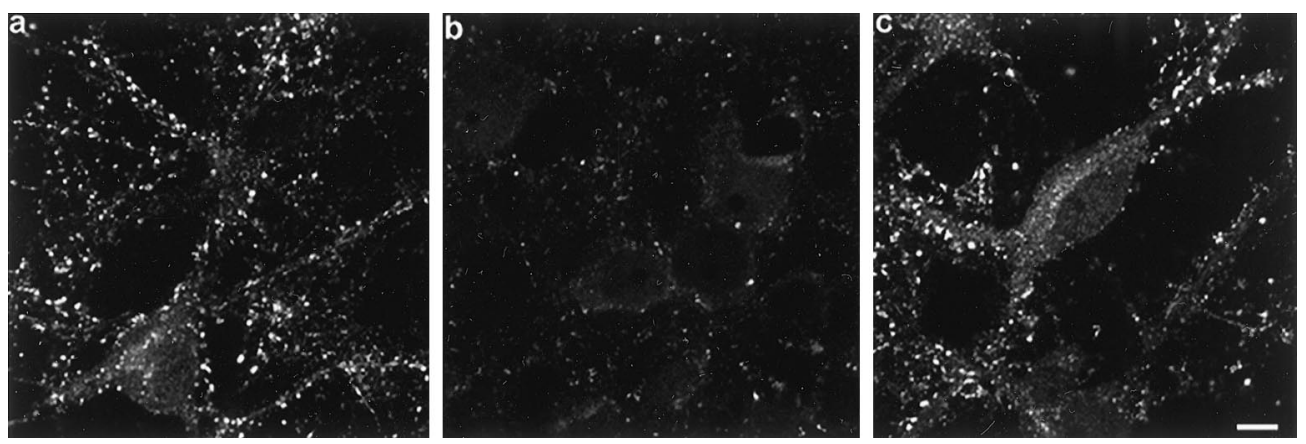
a
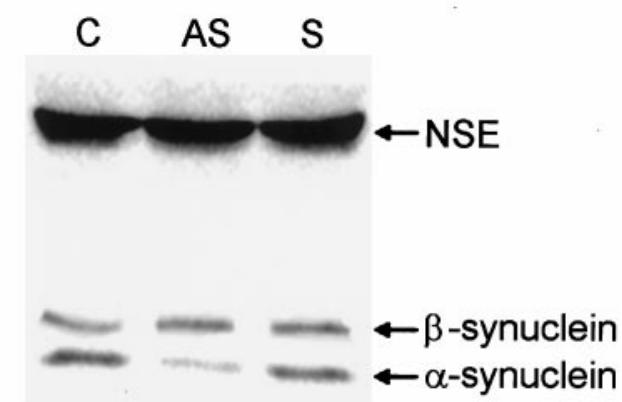

b

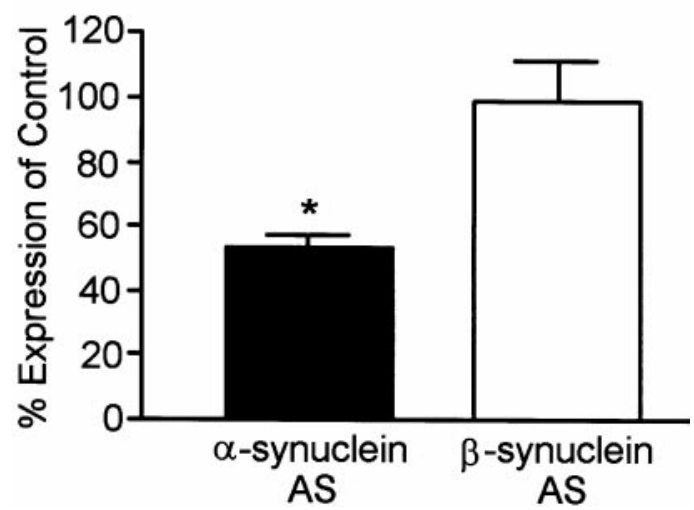

Figure 4. AS oligonucleotide treatment effectively decreases $\alpha$-synuclein protein expression. $a$, Quantitative Western blot analysis of $\alpha$-synuclein and NSE levels in control $(C)$, antisense $(A S)$-treated, and sense $(S)$ treated cultures for $6 \mathrm{~d}$. $b$, Quantitation of $\alpha$ - and $\beta$-synuclein levels in AS-treated cultures compared to control (untreated) cells. No differences were found between control and sense oligonucleotide-treated cultures. The closed bar represents $\alpha$-synuclein expression, and the open bar represents $\beta$-synuclein expression. Results are presented as the average percentage of expression of control cells \pm SEM. $n=3$; ${ }^{*} p<0.05$.

$\beta$-synuclein and NSE were unaffected (Fig. 4a,b). Treatment of the cells with a sense or scrambled oligonucleotide had no effect on the level of expression of $\alpha$-synuclein (Fig. $4 a$; data not shown). Although $\alpha$ - and $\beta$-synuclein exhibit sequence homology, the $\alpha$-synuclein-specific AS oligonucleotide had little effect on $\beta$-synuclein expression.

Cultures were also assessed for other proteins found in the presynaptic terminal to determine if they were affected by $\alpha$-synuclein suppression. The immunofluorescence localization of syntaxin, synaptophysin, synapsin I, and synaptobrevin, or vesicle-associated membrane protein (VAMP) showed little or no change after AS oligonucleotide treatment (Fig. 5). However, the staining intensity of vesicular boutons appeared to be slightly decreased for synapsin I (Fig. 5, compare $a, b$ ) and synaptophysin (Fig. 5, compare $c, d$ ). Examination of the levels of these synaptic proteins by quantitative Western analysis showed that synaptophysin and synapsin I indeed decreased in the AS oligonucleotide-treated cells $(68 \pm 10.1 \%$ and $63 \pm 9.7 \%$ of control, respectively), whereas VAMP, syntaxin, and NSE showed no significant change (Fig. 6).

To determine the ultrastructural consequences of $\alpha$-synuclein depletion on the presynaptic terminal, we compared the morphological characteristics of synapses from control, AS, and S oligonucleotide-treated cultures using electron microscopy. Ran-
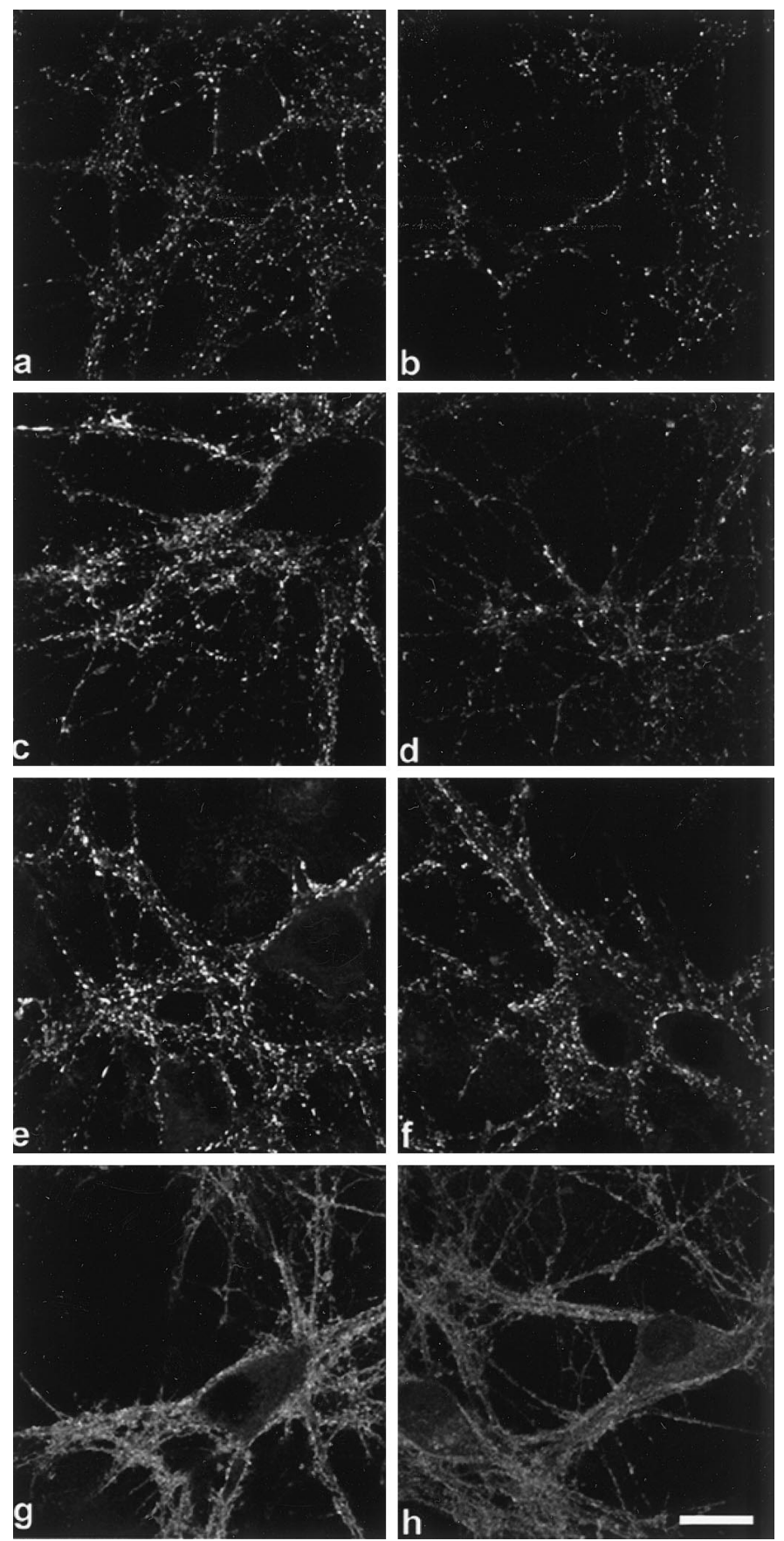

Figure 5. AS treatment results in an overall decrease of several presynaptic proteins. Left panels $(a, c, e, g)$ are control cultures, right panels $(b$, $d, f, h)$ are AS oligonucleotide-treated cultures. Synapsin I $(a, b)$, synaptophysin $(c, d)$, VAMP $(e, f)$, and syntaxin $(g, h)$. Although diminished fluorescent puncta were observed in $b$ and $d$, no changes in the localization of these presynaptic proteins were detected. Scale bar, $10 \mu \mathrm{m}$.

dom synapses that had readily observable postsynaptic densities were photographed at $33,000 \times$, and the vesicles were counted in each presynapse. No distinction was made between symmetrical or asymmetrical synapses, because $\alpha$-synuclein is found in both excitatory and inhibitory terminals, and discrimination of synapse type in cultured neurons is somewhat unreliable. Samples were pooled from four different cultures grown from different animals. Control values were comparable to those described for primary 


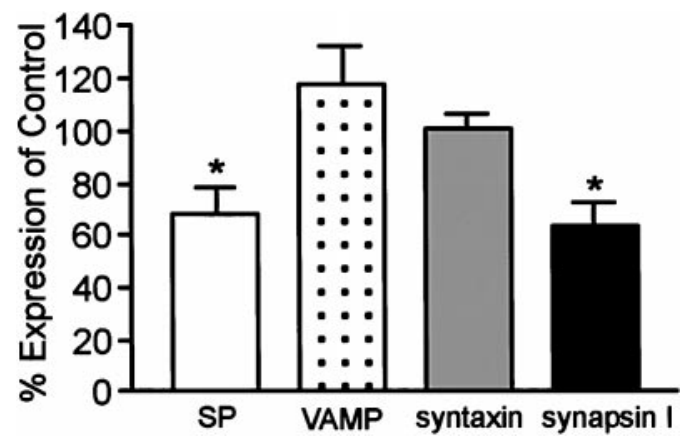

Figure 6. Effect of $\alpha$-synuclein AS oligonucleotide treatment on the expression of synaptic proteins. Quantitative Western blot analysis of synaptophysin, VAMP, syntaxin, and synapsin I levels in AS-treated cultures. All protein signals were normalized to the signal for NSE to account for the amount of neuronal protein loaded per lane. The open bar, dotted bar, gray bar, and black bar represent synaptophysin (SP) expression, VAMP expression, syntaxin expression, and synapsin I expression, respectively. Results are presented as the average percentage of expression of control cells \pm SEM. $n=3 ;{ }^{*} p<0.05$.

hippocampal neurons (Harris and Sultan, 1995; Schikorski and Stevens, 1997; Boyer et al., 1998). While the number of "docked" vesicles did not differ between the groups, the "distal" synaptic vesicle pool size was significantly reduced in the AS oligonucleotide-treated group as compared to the controls (Fig. 7). Statistical analysis of the vesicle numbers per square micrometer of synaptic area indicated that AS oligonucleotide-treated cultures had significantly fewer distal vesicles, whereas the docked vesicles in this group were unchanged (Table 1). The number of vesicles per square micrometer of synaptic area in $S$ oligonucleotide-treated cells was somewhat higher than that in control cultures.

\section{DISCUSSION}

It is now widely recognized that $\alpha$-synuclein plays an important role in the pathogenesis of several neurodegenerative diseases known collectively as synucleinopathies (Trojanowski et al., 1998). For example, $\alpha$-synuclein is found in Lewy bodies and neuronal fibrous cytoplasmic inclusions, as well as in Lewy neurites and dystrophic neuronal processes in brains of PD, DLB, and LBVAD patients (Spillantini et al., 1997; Baba et al., 1998). Also, $\alpha$-synuclein is likely the major component of glial and neuronal inclusions in multiple system atrophy (MSA) and Hallervorden-Spatz disease (Tu et al., 1998). The importance of $\alpha$-synuclein lesions in the pathogenesis of synucleinopathies is supported by the recent observations that $\alpha$-synuclein gene mutations are autosomally dominant in a subset of familial PD pedigrees (Polymeropoulos et al., 1997; Kruger et al., 1998). In addition, mutated $\alpha$-synuclein, as well as wild-type, aggregates into filaments in vitro (Conway et al., 1998; Crowther et al., 1998; El-Agnaf et al., 1998; Giasson et al., 1999). Despite our knowledge of these synuclein pathologies, little is known about the normal function of $\alpha$-synuclein in the mammalian brain, and we addressed this issue in the studies described here.

Although $\alpha$ - and $\beta$-synuclein were expressed almost exclusively at presynaptic terminals in mature primary neurons, both showed a delayed onset of expression and localization as compared to other presynaptic proteins. Our data are consistent with a previous study in which the expression of $\alpha$-synuclein was delayed in comparison to that of synapsin I in low-density cultures of rat hippocampal neurons (Withers et al., 1998). However, this previ-

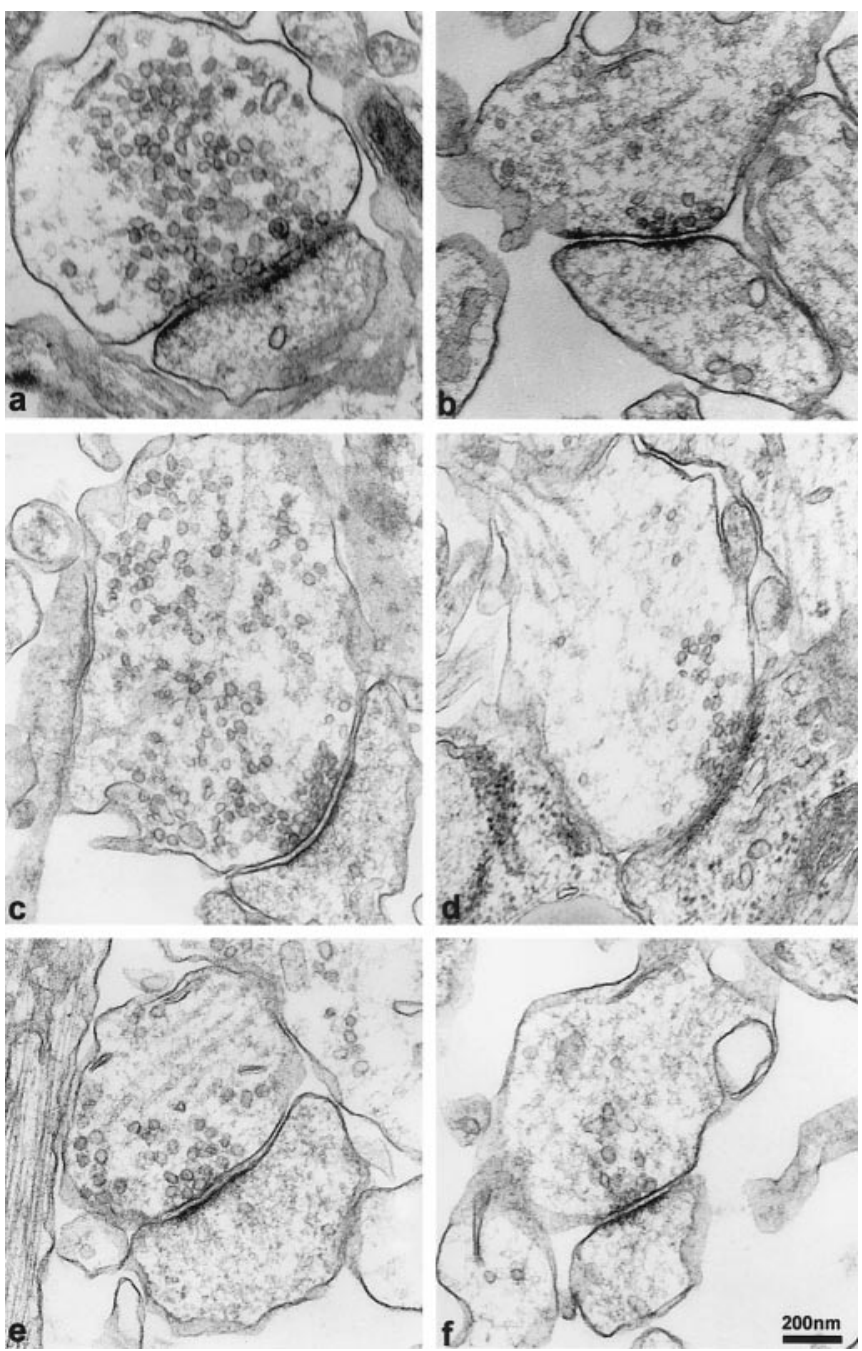

Figure 7. $\alpha$-Synuclein AS oligonucleotide treatment decreases the synaptic vesicle pool at the presynapse. Left panels $(a, c, e)$ are representative images of synapses from control hippocampal neurons. Right panels $(b, d$, $f$ ) are images of representative synapses from antisense oligonucleotidetreated hippocampal neurons. Note that synapses from both groups have a similar number of docked vesicles and possess well-defined postsynaptic densities. However, vesicles distal to the docking region are depleted in the AS oligonucleotide-treated cells (compare $a, c$, e with $b, d, f$ ). Scale bar, $200 \mathrm{~nm}$.

ous study showed a dramatic increase in $\alpha$-synuclein expression in 30 div cultures when compared with 5 div cultures, whereas we demonstrated here that the level of $\alpha$-synuclein expression peaks at 1 week and declines thereafter. These differences may be attributable to the fact that low-density cultures often form autaptic connections and synaptic development may differ from that of the high-density cultures used in our study. Here, it was shown that $\alpha$ - and $\beta$-synuclein expression at the synapse followed that of

Table 1. Vesicle counts per square micrometer of synaptic surface area

\begin{tabular}{llll} 
& Distal & Docked & Total \\
\hline Control & $64.9 \pm 3.3$ & $7.7 \pm 0.8$ & $72.4 \pm 3.3$ \\
Antisense & $38.7 \pm 2.8^{*}$ & $8.5 \pm 0.7$ & $47.6 \pm 3.2^{*}$ \\
Sense & $86.7 \pm 5.2$ & $9.2 \pm 0.9$ & $95.9 \pm 5.6$
\end{tabular}

${ }^{*} p<0.0001$. 
synaptophysin by at least a day. Whereas $\alpha$-synuclein levels were higher at 1 week versus later time points and $\beta$-synuclein levels did not significantly change over the culture period, both isoforms showed the same delayed localization to synapses and eventually became exclusively expressed at fully mature presynaptic terminals. This would suggest that these synucleins may function in the maintenance, rather than in the initial formation, of synapses. The lack of gross morphological deficits in the brains of mice lacking $\alpha$-synuclein further indicates that $\alpha$-synuclein is not essential for neuronal development and differentiation (Abeliovich et al., 2000).

To gain insight into the function of $\alpha$-synuclein, we used a specific AS oligonucleotide to decrease $\alpha$-synuclein expression in high-density primary hippocampal neurons that exhibit a robust development of well characterized synapses (Papa et al., 1995). By using an AS oligonucleotide in culture, we avoid some of the pitfalls experienced with genetic knock-outs that frequently do not demonstrate overt phenotypic changes (Janz and Sudhof, 1995). The AS oligonucleotide was successful in that it decreased $\alpha$-synuclein levels by $\sim 50 \%$, whereas $\beta$-synuclein levels remained unchanged. Because MAP2 staining was also unchanged, the AS oligonucleotide treatment did not adversely affect dendritic outgrowth. Moreover, $\alpha$-synuclein AS oligonucleotide treatment had the most dramatic effects when given repeatedly for several days, suggesting that presynaptic $\alpha$-synuclein may be long-lived. However, when $\alpha$-synuclein expression was blocked shortly after neurons were plated, this did not prevent the formation of synaptophysin-immunoreactive boutons (data not shown), again suggesting a role for $\alpha$-synuclein in synaptic maintenance rather than in the initiation of synapse formation.

If $\alpha$-synuclein is involved in the maintenance of fully functional synapses then a prolonged reduction in $\alpha$-synuclein levels may cause changes in presynaptic morphology. Indeed, we detected a decrease in the staining intensity at individual presynaptic boutons for synapsin I and synaptophysin which was accompanied by a decrease in their protein expression on quantitative Western blots. However, not all synaptic proteins were downregulated. For example, the levels of synaptobrevin (VAMP) and syntaxin did not decrease significantly. Since syntaxin is associated with the plasma membrane whereas synapsin I and synaptophysin are associated with synaptic vesicles (Sudhof, 1995), there may be differential consequences of $\alpha$-synuclein reduction on several structures at the synapses. However, the significance of the lack of a reduction in VAMP, also a synaptic vesicle protein, is unclear at this time. Analysis of the distribution and level of expression of presynaptic proteins in the $\alpha$-synuclein knockout mice does not show decreased expression of synaptophysin and synapsin I as we saw in the AS oligonucleotide-treated hippocampal cultures, although this was determined by immunostaining brain sections and therefore is not quantitative (Abeliovich et al. 2000).

Significantly, our ultrastructural analysis of presynaptic morphology and vesicles support a decrease in synaptic vesicles. Although the number of vesicles docked at the synaptic plasma membrane was unchanged, AS oligonucleotide-treated cultures displayed a marked reduction in the number of vesicles present in the distal pool, suggesting a possible role for $\alpha$-synuclein in the regulation of the store of vesicles available for transmitter release. Our EM results are not consistent with those from the $\alpha$-synuclein knock-out mice, which do not show any difference in synaptic vesicles (Abeliovich et al. 2000). However, vesicles were not counted and a statistical analysis was not performed in the knockout mice. Additionally, the differences could be caused by the analysis of neurons from different brain regions (hippocampus vs striatum) or the age of the neurons (embryonic vs adult).

Our results are consistent with a role for $\alpha$-synuclein in the maintenance of previously existing mature synapses and the stabilization of synaptic function. There is much evidence to suggest that the distal pool of synaptic vesicles is anchored by the actin cytoskeleton in the presynaptic terminal, and numerous studies have demonstrated that this binding is mediated by synapsin I (for review, see Greengard et al., 1994). Synapsin I, which is peripherally associated with the vesicle membrane, can bind vesicles to actin in a phosphorylation-dependent manner (Stefani et al., 1997), and $\alpha$-synuclein may potentiate or stabilize this interaction. $\alpha$-Synuclein has been shown to bind to artificial phospholipid membranes (Davidson et al., 1998) as well as to synaptic vesicles (Jensen et al., 1998), which would support a vesicleanchoring function. It is known that synapsin I is phosphorylated by CaMKII (Benfenati et al., 1992) present on presynaptic vesicles and $\beta$-synuclein is also phosphorylated on serine residues by CaMKII (Nakajo et al., 1993). Therefore, perhaps $\alpha$-synuclein is also phosphorylated by kinases present at the presynaptic terminal. Furthermore, a significant fraction of synapsin I is transported via slow component b (Greengard et al., 1993), which may also be the means of transport for $\alpha$-synuclein (Jensen et al., 1998). Thus, similarities exist between synapsin I and the synucleins, suggesting they may regulate vesicles in a similar manner. Alternatively, $\alpha$-synuclein may modulate the expression, regulation, or activity of synapsin I itself. Therefore, alterations in $\alpha$-synuclein would likely affect the vesicular pool.

These observations have implications relevant to mechanisms of $\alpha$-synuclein pathologies and their role in PD and AD because our data suggest that $\alpha$-synuclein is a predominantly presynaptic protein involved in the maintenance of synaptic vesicle pools in primary neurons. It is known that the familial PD mutations in the $\alpha$-synuclein gene can abolish the binding of $\alpha$-synuclein to presynaptic vesicles (Jensen et al., 1998) and that $\alpha$-synuclein forms Lewy bodies in sporadic PD. Thus, the data presented here may signify that the availability of vesicles for release in familial and sporadic PD brains could be compromised by $\alpha$-synuclein pathologies, thereby leading to impaired synaptic function and degeneration.

\section{REFERENCES}

Abeliovich A, Schmitz Y, Fariñas I, Choi-Lundberg D, Ho W-H, Castillo PE, Shinsky N, Verdugo JMG, Armanini M, Ryan A, Hynes M, Philips H, Sulzer D, Rosenthal A (2000) Mice lacking $\alpha$-synuclein display functional deficits in the nigrostriatal dopamine system. Neuron 25:239-252.

Baba M, Nakajo S, Tu P, Tomita T, Nakaya K, Lee VM-Y, Trojanowski JQ, Iwatsubo I (1998) Aggregation of $\alpha$-synuclein in Lewy bodies of sporadic Parkinson's disease and dementia with Lewy bodies. Am J Pathol 152:879-884.

Banker G, Goslin K (1991) Culturing nerve cells. Cambridge, MA, MIT.

Bartlett WP, Banker G (1984) An electron microscopic study of the development of axons and dendrites by hippocampal neurons in culture. II. Synaptic relationships. J Neurosci 4:1954-1965.

Benfenati F, Valtorta F, Rubenstein JL, Gorelick FS, Greengard P, Czernik AJ (1992) Synaptic vesicle-associated $\mathrm{Ca}^{2+} /$ calmodulindependent protein kinase II is a binding protein for synapsin I. Nature 359:417-420.

Boyer C, Schikorski T, Stevens CF (1998) Comparison of hippocampal dendritic spines in culture and in brain. J Neurosci 18:5294-5300.

Buchman VL, Hunter HJA, Pinõn LGP, Thompson J, Privalova EM, Ninkina NN, Davies AM (1998) Persyn, a member of the synuclein 
family, has a distinct pattern of expression in the developing nervous system. J Neurosci 18:9335-9341.

Conway KA, Harper JD, Lansbury PT (1998) Accelerated in vitro fibril formation by a mutant alpha-synuclein linked to early-onset Parkinson disease. Nat Med 4:1318-1320.

Crowther RA, Jakes R, Spillantini MG, Goedert M (1998) Synthetic filaments assembled from C-terminally truncated alpha-synuclein. FEBS Lett 436:309-312.

Davidson WS, Jonas A, Clayton DF, George JM (1998) Stabilization of alpha-synuclein secondary structure upon binding to synthetic membranes. J Biol Chem 273:9443-9449.

El-Agnaf OMA, Jakes R, Curran MD, Wallace A (1998) Effects of the mutations Ala ${ }^{30}$ to Pro and Ala ${ }^{53}$ to Thr on the physical and morphological properties of $\alpha$-synuclein protein implicated in Parkinson's disease. FEBS Lett 440:67-70.

Giasson BI, Jakes R, Goedert M, Duda JE, Leight S, Trojanowski JQ, Lee VMY (2000) A panel of epitope specific antibodies detects protein domains distributed throughout human $\alpha$-synuclein in Lewy bodies of Parkinson's disease. J Neruosci Res 59:528-533.

Giasson BI, Uryu K, Trojanowski JQ, Lee VMY (1999) Mutant and wild type human alpha-synucleins assemble into elongated filaments with distinct morphologies in vitro. J Biol Chem 274:7619-7622.

Greengard P, Valtorta F, Czernik AJ, Benfenati F (1993) Synaptic vesicle phosphoproteins and regulation of synaptic function. Science 259:780-785.

Greengard P, Benfenati F, Valtorta F (1994) Synapsin I, an actinbinding protein regulating synaptic vesicle traffic in the nerve terminal. Adv Second Messenger Phosphoprotein Res 29:31-45.

Harris KM, Sultan P (1995) Variations in the number, location and size of synaptic vesicles provides an anatomical basis for the nonuniform probability of release at hippocampal CA1 synapses. Neuropharmacology 34:1387-1395.

Hsu LJ, Mallory M, Xia Y, Veinbergs I, Hashimoto M, Yoshimoto M, Thal LJ, Saitoh T, Masliah E (1998) Expression pattern of synucleins (non-Abeta component of Alzheimer's disease amyloid precursor protein/alpha-synuclein) during murine brain development. J Neurochem 71:338-344.

Irizarry MC, Kim T-W, McNamara M, Tanzi RE, George JM, Clayton DF, Hyman BT (1996) Characterization of the precursor protein of the non- $\mathrm{A} \beta$ component of senile plaques (NAcP) in the human central nervous system. J Neuropathol Exp Neurol 55:889-895.

Jakes R, Spilantini MG, Goedert M (1994) Identification of two distinct synucleins from human brain. FEBS Lett 345:27-32.

Janz R, Sudhof TC (1995) A systematic approach to studying synaptic function in vertebrates. Cold Spring Harb Symp Quant Biol 60:309-314.

Jensen PH, Nielsen MS, Jakes R, Dotti CG, Goedert M (1998) Binding of alpha-synuclein to brain vesicles is abolished by familial Parkinson's Disease mutation. J Biol Chem 273:26292-26294.

Ji H, Liu YE, Jia T, Wang M, Liu J, Xiao G, Joseph BK, Rosen C, Shi YE (1997) Identification of a breast cancer-specific gene, BCSG1, by differential cDNA sequencing. Cancer Res 57:759-764.

Kruger R, Kuhn WMT, Woitaqlla D, Greaber M, Kosel S, Pruntek H,
Epplen JT, Schols L, Reiss O (1998) Ala30Pro mutation in the gene encoding $\alpha$-synuclein in Parkinson's disease. Nat Genet 18:106-108.

Maroteaux L, Campanelli JT, Scheller RH (1988) Synuclein: a neuronspecific protein localized to the nucleus and presynaptic nerve terminal. J Neurosci 8:2804-2815.

Nakajo SK, Omata T, Aiuchi T, Shibayama I, Okahashi H, Ochiai Y, Nakai K, Nakaya K, Nakamura Y (1990) Purification and characterization of a novel brain-specific $14-\mathrm{kDa}$ protein. J Neurochem 55:2031-2038.

Nakajo S, Tsukada K, Omata K, Nakamura Y, Nakaya K (1993) A new brain-specific $14-\mathrm{kDa}$ protein is a phosphoprotein. Its complete amino acid sequence and evidence for phosphorylation. Eur J Biochem 217:1057-1063.

Papa M, Bundman MC, Greenberger V, Segal M (1995) Morphological analysis of dendritic spine development in primary cultures of hippocampal neurons. J Neurosci 15:1-11.

Polymeropoulos MH, Lavedan C, Leroy E, Ide SE, Dehejia A, Dutra A, Pike B, Root H, Rubenstein J, Boyer R, Stenroos ES, Chandrasekharappa S, Athanassiadou A, Papapetropoulos T, Johnson WG, Lazzarini AM, Duvoisin RC, Di Iorio G, Golbe LI, Nussbaum RL (1997) Mutation in the alpha-synuclein gene identified in families with Parkinson's disease. Science 276:2045-2047.

Pozzo-Miller LD, Gottschalk W, Zhang L, McDermott K, Du J, Gopalakrishnan R, Oho C, Sheng ZH, Lu B (1999) Impairments in high-frequency transmission, synaptic vesicle docking, and synaptic protein distribution in the hippocampus of BDNF knockout mice. J Neurosci 19:4972-4983.

Reist NE, Buchanan J, Li J, DiAntonio A, Buxton EM, Schwarz TL (1998) Morphologically docked synaptic vesicles are reduced in synaptotagmin mutants of Drosophila. J Neurosci 18:7662-7673.

Schikorski T, Stevens CF (1997) Quantitative ultrastructural analysis of hippocampal excitatory synapses. J Neurosci 17:5858-5867.

Spillantini MG, Schmidt ML, Lee VM-Y, Trojanowski JQ, Jakes R, Goedert M (1997) Alpha-synuclein in Lewy bodies. Nature 388:839-840.

Stefani G, Onofri F, Valtorta F, Vaccaro P, Greengard P, Benfenati F (1997) Kinetic analysis of the phosphorylation-dependent interactions of synapsin I with rat brain synaptic vesicles. J Physiol (Lond) 504:501-515.

Sudhof T (1995) The synaptic vesicle cycle: a cascade of protein-protein interactions. Nature 375:645-653.

Surguchov A, Surgucheva I, Solessio E, Bashr W (1999) Synoretin-a new protein belonging to the synuclein family. Mol Cell Neurosci 13:95-103.

Trojanowski JQ, Goedert M, Iwatsubo T, Lee VM-Y (1998) Fatal attractions: abnormal protein aggregation and neuron death in Parkinson's Disease and Lewy Body dementia. Cell Death Diff 5:832-837.

Tu P, Galvin J, Baba M, Giasson B, Tomita T, Leight S, Nakajo S, Iwatsubo T, Trojanowski J, Lee VMY (1998) Glial cytoplasmic inclusion in white matter oligodendrocytes of multiple system atrophy brains contain insoluble $\alpha$-synuclein. Ann Neurol 44:415-422.

Withers GS, George JM, Banker GA, Clayton DF (1997) Delayed localization of synelfin (synuclein, NACP) to presynaptic terminals in cultured rat hippocampal neurons. Brain Res Dev Brain Res 99:87-94. 